\title{
Dynamic fluorescence imaging of the free radical products of X-ray absorption in live cells
}

\author{
Catherine A Rappole ${ }^{1}$, Kasturi Mitra ${ }^{2}$ and Han Wen ${ }^{1 *}$
}

\begin{abstract}
The immediate products of $\mathrm{x}$-ray absorption in aqueous biological samples are free radicals including ${ }^{*} \mathrm{OH}, \mathrm{H}_{2} \mathrm{O}_{2},{ }^{*} \mathrm{H}$ and solvated electrons. Because their lifetimes and diffusion ranges are dependent on the local bio-molecular environment, imaging these free radicals in real-time while they are produced by a scanning $x$-ray nanobeam may provide a biological microscopy method of high resolution. As a first step towards this goal, we investigated the feasibility of imaging the initial free radical products of $x$-ray absorption in live cells using fluorescent free radical sensors. We selected six commercially available fluorescent sensors for screening tests of their sensitivities towards $x$-ray radiation in solution form. Two of the six dyes were found to have high sensitivities. One of the two was successfully used for dynamic confocal fluorescence imaging of $x$-ray generated free radicals in the intracellular space of mouse embryonic fibroblasts. Time series of fluorescence images before and during $x$-ray radiation were acquired. The rate of increase of cellular fluorescence showed both the initial production of free radicals by the physical ionization events as well as stimulated biological production of reactive oxygen species later on. The implications of the results for future development of microscopy techniques are discussed.
\end{abstract}

Keywords: X-ray activated, Free radical, Reactive oxygen species, ROS sensor, Fluorescence imaging, Microscopy, Scanning nanobeam

\section{Background}

The absorption of $\mathrm{x}$-ray photons in aqueous biological samples results in the ionization of water molecules into free radical products, which react with biomolecules in the local environment and begin a cascade of long lasting biological effects(Schmidt-Ullrich et al. 2000; Spitz et al. 2004). The purpose of our study is to image the immediate free radical products of the $\mathrm{x}$-ray ionization events in live cells in situ using fluorescent sensors. Fluorescent sensors of reactive oxygen species (ROS) have been used effectively to assay radiation-induced free radicals and oxidative stresses in cells(Morales et al. 1998; Narayanan et al. 1997; Wan et al. 2003). They are non-fluorescent molecules in their initial state and are converted to fluorescent forms upon reaction with free radicals. Over a decade ago Leach and co-authors demonstrated elegantly that fluorescence microscopy of the ROS sensor Dihydro-DCF can monitor radiation-induced, mitochondria-dependent generation of

\footnotetext{
* Correspondence: wenh@nhlbi.nih.gov

${ }^{1}$ Imaging Physics Lab, Biophysics and Biochemistry Center, National Heart, Lung and Blood Institute, National Institutes of Health, Bethesda, MD 20892, USA

Full list of author information is available at the end of the article
}

ROS in live cells(Leach et al. 2001). However, to date we are not aware of any studies that focus on imaging the initial free radical products of $\mathrm{x}$-ray absorption.

Our interest in imaging the initial free radical products of $x$-ray absorption in situ is prompted by the rapid progress of intense $\mathrm{x}$-ray nanobeams at synchrotron sources (Cholewa et al. 2001; Ice et al. 2011; Kang et al. 2006; Mimura et al. 2010)and possibly in laboratory settings (Kneip et al. 2010), which can potentially deliver bursts of free radicals to specific sites in a cell or tissue at nanometer resolution(Prise and Schettino 2011). These free radicals have limited lifetimes and diffusion ranges (a few nanometers for the most abundant hydroxyl free radical, for example)(Kim 2008; Roots and Okada 1975). They are therefore localized to the site of irradiation.

With this in mind, fluorescence imaging of the initial free radical products in real time may allow a biological imaging method to be developed, where an $\mathrm{x}$-ray nanobeam is scanned over the sample to achieve high resolution, and simultaneously the immediate radiolysis products are detected by imaging the fluorescent sensors. The fluorescence signal will be dependent on the 
lifetime and mobility of the initial free radicals, which in turn relates to their interaction with biomolecules at the site of irradiation(Roots and Okada 1975). Therefore, the combination of free radical imaging and $\mathrm{x}$-ray nanobeam scanning may provide biological information at high resolution. Another potential use of this combination is to image immobilized free radical sensor molecules at high resolution. If such sensor molecules can be attached to structures of interest, then they function as $\mathrm{x}$-ray activated fluorescent tags in analogy to photoactivated fluorescent dyes(Betzig et al. 2006; Patterson and Lippincott-Schwartz 2002).

As a first step towards these goals, we aim to perform in situ fluorescence imaging of free radical production in cells under the irradiation of a compact $x$-ray tube. In aqueous biological samples, $\mathrm{x}$-ray absorption is predominantly by water molecules. The primary free radical products of the radiolysis of water and their yields (molecules/100 eV) are $\cdot \mathrm{OH}(2.80)$, e $\mathrm{e}_{\mathrm{aq}}^{-}(2.65), \mathrm{H}_{2} \mathrm{O}_{2}$ (0.68), and $\cdot \mathrm{H}(0.60)$ (Meesungnoen et al. 2001; Sehested et al. 1973). There are many commercially available fluorescent ROS sensors that may respond to one or more of the above products. Generally upon reaction with the free radicals, the dye molecule is transformed from a non-fluorescent to a fluorescent form.

We selected an initial set of 6 fluorescent ROS sensors for sensitivity measurements in solution state against $\mathrm{x}$ ray irradiation. Based on the results, one of the 6 dyes was further tested in live cell imaging experiments. Our basic experimental design was to observe the fluorescence in the cells over a period of time before the $x$-ray source was turned on, which served as the control. Then the $\mathrm{x}$-ray beam was switched-on to produce free radicals continuously and cause a steady rise of the fluorescence level. The rate of fluorescence increase represents the rate of conversion of the ROS sensor, which in turn reflects the rate of free radical production in the cells.

\section{Methods}

Initial selection of fluorescent ROS sensors Selection criteria

The selection of fluorescent sensors for this study was based on published literature, especially the review articles by P. Wardman(Wardman 2007) and by A. Gomes and co-authors(Gomes et al. 2005) and the study on fluorescence probes of hydroxyl radical by Cohn and co-authors (Cohn et al. 2008). For initial testing of sensitivity towards $\mathrm{x}$-ray radiation in solution form, we used the following inclusion criteria to select the fluorescent dyes : 1 . The dye is commercially available; 2 . It is directly activated by one or more of the free radical products of water radiolysis without the need for an intermediary; 3 . To ensure that we observe free radical production in the intracellular volume, the pre-activated form can diffuse into intracellular space; 4. Both the pre-activated and activated forms should be sequestered in the intracellular space and not leak out into the extracellular medium.

Based on these criteria, six fluorescent dyes were included in the initial sensitivity measurements against $x$ ray radiation. A list of their names, abbreviations, peak excitation/emission wavelengths, and the fluorescent forms upon reaction with free radicals is given in Table 1.

All six dyes come in reduced, non-fluorescent forms. Upon oxidation by free radicals, they are converted to stable fluorescent forms that can be detected by fluorescence spectrometry and microscopy. Since the initial non-fluorescent forms are susceptible to oxidation by UV light and spontaneous free radical formation in solutions, it was necessary for us to do all experimental procedures under low, red lights and avoid prolonged storage of the dyes in solution form.

\section{Fluorescence spectrometer measurements}

The sensitivity measurements were performed in solution using a Jobin Yvon fluorescence spectrometer (Model FL-1039, HORIBA Scientific) or a laser scan fluorescence microscope (Zeiss LSM410, Carl Zeiss Microscopy Inc.). The $\mathrm{x}$-ray source was a compact tungsten anode tube (SB-80-500, Source-Ray Inc.) operating at 20 Watts $/ 80 \mathrm{kVp} / 0.5 \mathrm{~mA}$. Dye solutions of $100 \mu \mathrm{M}$ concentration were prepared in the appropriate buffers (Additional file 1).

For the spectrometer measurements, each dye solution was exposed to three 10 -second periods of $x$-ray radiation, corresponding to accumulated doses of $0.5,1.0$ and 1.5 Gy. Fluorescence emission spectra were acquired

Table 1 Fluorescent ROS sensors included in initial sensitivity measurements towards x-ray radiation

\begin{tabular}{llll}
\hline Name & Abbreviation & Peak excitation/Emission wavelength (nm) & Fluorescent form \\
\hline 2',7'-dihydrodichlorofluorescein diacetate $^{\prime}$ & $\mathrm{H}_{2}$ DCFDA & $501 / 521$ & Dichlorofluorescein \\
Dihydrofluorescein & DHF & $494 / 521$ & Fluorescein \\
Dihydrorhodamine 123 & DHR-123 & $507 / 529$ & Rhodamine 123 \\
Aminophenyl Fluorescein & APF & $494 / 521$ & Fluorescein \\
Hydroethidine & HE & $473 / 610$ & Ethidium \\
Dihydroresorufin & Amplex Red & $571 / 585$ & Resorufin \\
\hline
\end{tabular}


before the $\mathrm{x}$-ray exposure and after each exposure period. Fluorescence intensity was calculated as the sum of the intensity under the emission peak. The amount of fluorescence increase per Gy of radiation was quantified by linear regression of the fluorescence intensity vs. dose curve. For the purpose of comparing dyes of different emission spectra, we measured the fluorescence intensity of the oxidized (fluorescent) form of each dye at the same concentration. The fluorescent forms of the dyes are given in Table 1.

Finally, the normalized sensitivity of each fluorescent sensor to $\mathrm{x}$-ray irradiation was defined as the percentage of the non-fluorescent form of the dye that are converted to the fluorescent form by the exposure dose of 1 Gy. This quantity is given by the ratio (Fluorescence increase per Gy)/(fluorescence of the fluorescent form of the dye). Since this value is dependent on the dye concentration in the solution, it was important to use the same concentration for all dyes.

Each spectrometer measurement was repeated three times in separate samples.

\section{Fluorescence microscopy measurements of Dye solutions}

The sensitivities of the fluorescent ROS sensors 2,7'dihydrodichlorofluorescein diacetate $\left(\mathrm{H}_{2}\right.$ DCFDA $)$ and dihydrofluorescein (DHF) were compared using a laser scanning fluorescence microscope (Zeiss LSM410). Tests were done in dye solutions of $100 \mu \mathrm{M}$ concentration. Since $\mathrm{H}_{2}$ DCFDA is not reactive to free radicals until the diacetate group is cleaved (by intracellular esterase enzymes for example), we used a chemical process to cleave the diacetate group when preparing the solution (see Additional file 1). The solutions were loaded into sample wells, which were made by attaching $0.1 \mathrm{~mm}$ thick double-adhesive ring spacers (Electron Microscopy Sciences) on No. 0 glass coverslips. Eight $\mu \mathrm{L}$ of solution was loaded into a well and sealed on top with another No. 0 coverslip, then placed on the microscope stage for imaging.

The microscope was a modified inverted laser scanning confocal fluorescence microscope (Figure 1A). The condenser optics were removed to allow the $\mathrm{x}$-ray tube to be placed over the sample stage. The distance between the focal spot of the $\mathrm{x}$-ray tube and the sample plane was $41 \mathrm{~mm}$. When the $\mathrm{x}$-ray tube operated at its maximum output of $80 \mathrm{kVp} / 0.5 \mathrm{~mA}$, the radiation dose rate on the sample was $3.0 \mathrm{~Gy} / \mathrm{min}$, as measured with a dosimeter (TANDEM dosimeter, PTW-Freiburg, Germany). The dosimeter was factory calibrated for $\mathrm{x}$-ray CT scanners operating at various generator input voltages. Specifically, we placed the air ionization chamber of the dosimeter at the same distance from the $\mathrm{x}$-ray source as the sample stage, and obtained a dose rate readout while running the $\mathrm{x}$-ray tube at the experimental condition. The readout represented the equivalent radiation absorption dose rate in typical soft tissue, which is the amount of absorbed energy per gram of tissue per minute $(1 \mathrm{~Gy} / \mathrm{min}$ is equivalent to 1 milli-joule/g/min). Since our aqueous samples had approximately the same density as soft tissue, the dosimeter readout was used for the radiation absorption dose rate in the sample. This dose rate was applied to all microscopy experiments, including solution samples and cell samples.

In each $\mathrm{x}$-ray test, a series of fluorescence images at $488 \mathrm{~nm}$ excitation were taken before and during $\mathrm{x}$-ray exposure. Each image took 1-2 seconds to acquire and the interval between images was 30 seconds. For each of the two fluorescent sensors, the experiment was repeated three times in separate samples.

\section{Cell imaging}

\section{Preparation of cell samples}

Based on the sensitivity measurements performed in solutions, one of the 6 fluorescent ROS sensors, $\mathrm{H}_{2}$ DCFDA, was chosen for cell imaging. Mouse embryonic fibroblast (MEF) cells were cultured in Dulbecco's Modified Eagle Medium (D-MEM) in $75 \mathrm{~cm}^{2}$ cell culture flasks (see Additional file 1). In preparation for an experiment the cells were seeded on glass-bottom dishes (35 mm diameter petri dish with $14 \mathrm{~mm}$ micro well of No. 0 coverglass bottom, MatTek Corporation) and incubated overnight (see Additional file 1). The glassbottom dishes are suitable for imaging on inverted microscopes. On the day of the experiment the dishes were removed from the incubator and confluency checked with a light microscope. The $\mathrm{H}_{2}$ DCFDA dye was loaded into the cells by the following procedure: the culture medium in the dishes was aspirated and the cells were washed with Dulbecco's phosphate-buffered saline (DPBS); freshly prepared $100 \mu \mathrm{M} \mathrm{H}_{2}$ DCFDA solution (see Additional file 1) was added to the dishes and incubated for 30 minutes; the dye solution was aspirated from the dishes, followed by washing with DPBS to remove residual dye in the medium; the cells were further incubated in DPBS buffer for 30 minutes to allow cellular esterases to hydrolyze the acetate groups and make the dye responsive to oxidation. The dishes were kept in dark condition to prevent photo-oxidation of the dye.

\section{Imaging free radical formation in cells under X-Ray}

The glass-bottom dishes containing the cells were loaded onto the stage of the modified confocal microscope (Figure 1A), directly under the window of the $\mathrm{x}$-ray tube. An issue that needed attention was the attenuation of the $x$-ray beam by the layer of buffer medium above the cells, as the cells adhered to the glass bottom of the dishes. A dish contained $1.5 \mathrm{~mL}$ of DPBS buffer, which amounted to a layer thickness of $2.0 \mathrm{~mm}$ over 


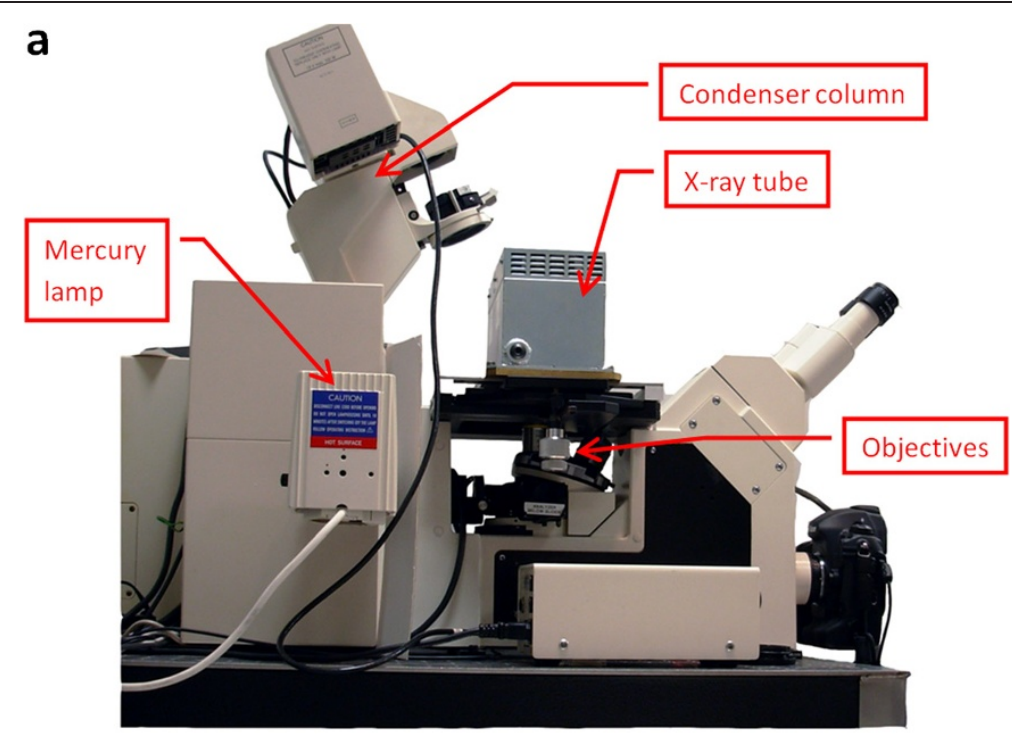

b
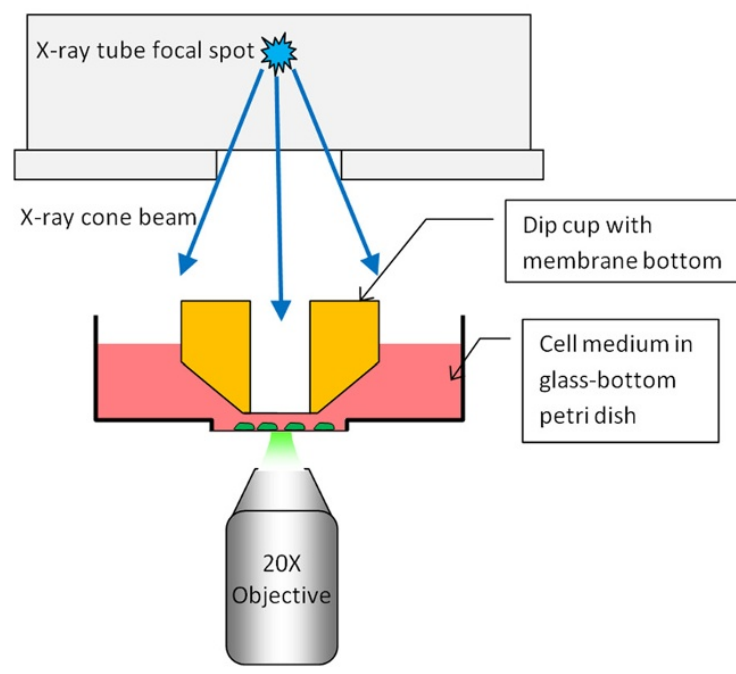

Figure 1 Illustration of the modified confocal fluorescence microscope for imaging x-ray generated free radicals in live cells. (a) The Zeiss LSM410 microscope was modified by removing the condenser optics and tilting back the condenser column (contact switch disabled) to allow the $x$-ray tube to be positioned over the sample stage. The microscope's mercury lamp was used for UV illumination. All cell experiments used a 20X objective. (b) The custom sample stage minimizes attenuation of the x-ray beam by the buffer medium above the cells while maintaining the volume of the medium. The stage consists of a glass-bottom petri dish suitable for inverted microscopes, and a dip cup above the glass bottom of the dish that serves as a window for the $x$-ray beam. The dip cup has a membrane bottom of $30 \mu m$ Kapton film. The layer of buffer medium between the membrane and the cells is $0.4 \mathrm{~mm}$.

the cells. The solution to this problem is shown in Figure 1B. An $x$-ray window was created above the micro well of the dishes using a dip cup with a membrane bottom. The dip cup was made of Teflon and the membrane was a $30 \mu \mathrm{m}$ layer of Kapton. The cup reduced the thickness of the buffer layer beneath the $\mathrm{x}$-ray window to $0.4 \mathrm{~mm}$, while maintaining contact between the cells and the bulk of the buffer medium.

Fluorescence imaging was performed at ambient $21^{\circ} \mathrm{C}$ temperature, with $488 \mathrm{~nm}$ laser excitation and $20 \mathrm{X}$ objective. The microscope was programmed to take fifteen images in a time series, with 30 seconds interval between each image. The $\mathrm{x}$-ray tube was switched on at 5 seconds after the fifth image, and remained on during the rest of image acquisition (5 minutes). Experiments on five separate samples were carried out. Four of the five samples contained sufficient number of cells in the field of view and were used for data processing.

The Image J software was used for data processing. The processing procedure included creating a mask of the cells and finding the average image intensity within the mask as a function of time. The cell mask was 
created in two steps. First the average of the first 5 images prior to $\mathrm{x}$-ray exposure was calculated as a before-exposure image. Then, the difference between the last image taken after 5 minutes of exposure and the before-exposure image was calculated. An intensity threshold was applied to the difference image, resulting in a mask that only contained the cells. The mask was then used to calculate the average fluorescence intensity in the cells for each image in the time series. We call this quantity the cellular fluorescence intensity.

Since $x$-ray induced free radical formation includes the initial, physical products of radiolysis and subsequent stimulated production of free radicals by the biological mechanisms of the cells (Leach et al. 2001; Spitz et al. 2004), we are interested in the change of the rate of free radical formation over the time course of radiation. For this purpose we calculated the rate of increase of the cellular fluorescence intensity, which was defined as (difference of cellular fluorescence intensity between two consecutive images)/(time interval between the two images). The rates at three time points were calculated before radiation, at the onset of radiation and at 5 minutes of radiation. The rate before radiation was calculated from the first 5 images before the $\mathrm{x}$-ray tube was switched on. The rate at the onset of radiation was calculated between images No. 5 and 6, during which time the cells received the first 1.3 Gy of radiation. The rate at 5 minutes of radiation was calculated between images No. 14 and 15, after the cells had received 13.3 Gy of radiation.

\section{Imaging free radical formation in cells from UV light}

As a reference for the above $\mathrm{x}$-ray experiments, we also observed free radical formation in cells that received 5

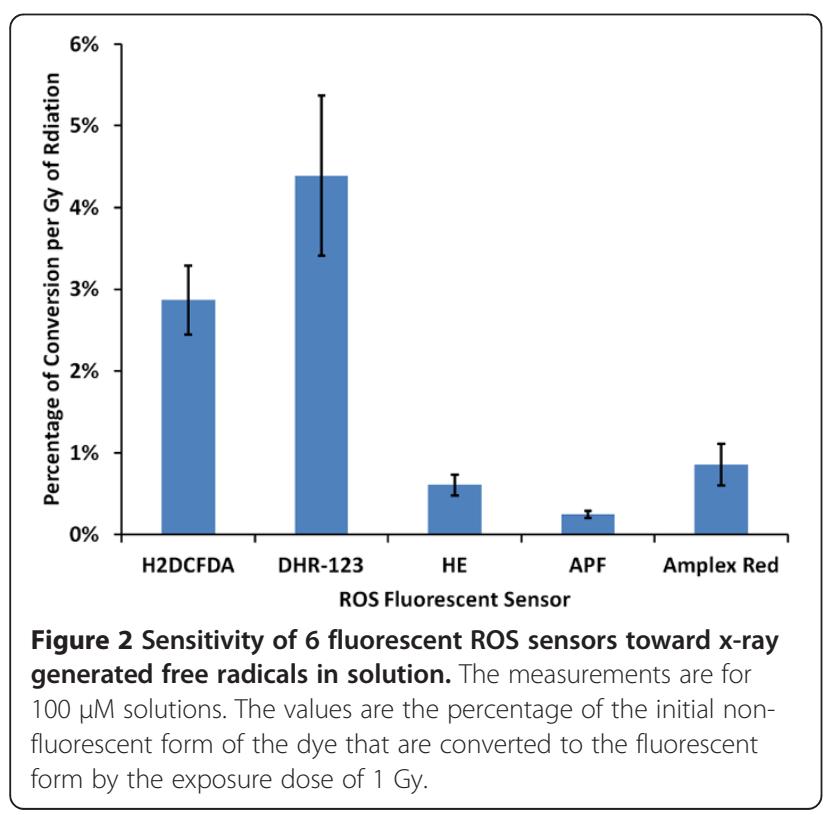

minutes of mercury lamp illumination. The cell samples were prepared following the same protocol as the $x$-ray experiments. A confocal fluorescence image was acquired immediately before the mercury lamp was switched on. Then the cells were illuminated for 5 minutes, and a

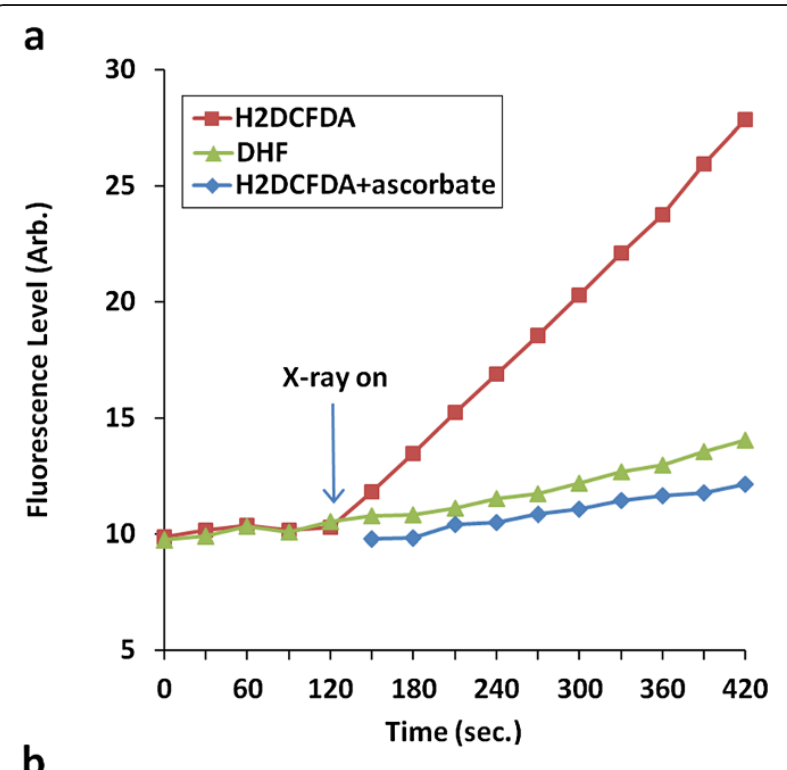

b

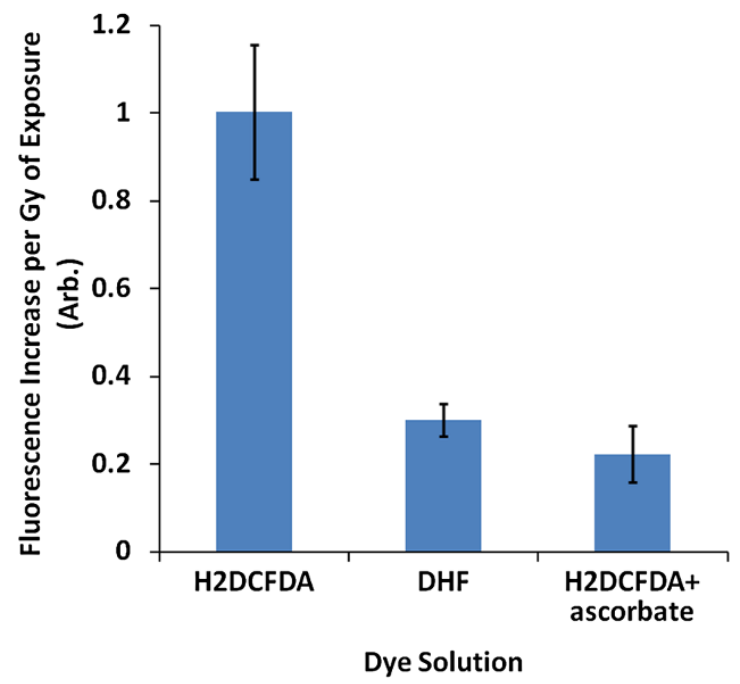

Figure $3 \mathrm{X}$-ray induced fluorescence increase in $100 \mu \mathrm{M}$ solutions of de-esterified H2DCFDA, DHF, and de-esterified H2DCFDA + $1 \mathrm{mM}$ ascorbate (free radical scavenger). (a) Time series of fluorescence images were acquired on the modified confocal microscope (Figure 1). The interval between images was 30 seconds. For the first two solutions the $x$-ray tube was switched on after image No.5, and persisted to the end of the time series. An immediate rise of the fluorescence intensity can be seen in the deesterified H2DCFDA solution, while a smaller response was seen in the DHF solution. For the de-esterified H2DCFDA + ascorbate solution the fluorescence over 5 minutes of $x$-ray exposure was recorded. (b) The average increase of fluorescence intensity per unit dose of $x$-ray exposure was calculated for the 3 solutions from 3 separate samples for each dye. The error bars are standard deviation. 
second fluorescence image was acquired. A total of 8 separate samples were studied.

For image processing, a cell mask was generated based thresholding the difference image before and after illumination, as described in the previous section. The mask was used to calculate the average increase of cellular fluorescence intensity from mercury lamp illumination.

\section{Results and discussion}

In the following sections all values are presented as mean \pm standard deviation.

\section{Comparison of sensitivity to X-Ray radiation in 6 fluorescent ROS sensors}

As described in the subsection of "Materials and Methods - Initial Selection of Fluorescent ROS Sensors Fluorescence Spectrometer Measurements", the normalized sensitivity is measured in $100 \mu \mathrm{M}$ dye solutions as the percentage of the non-fluorescent form of the dye that are converted to the fluorescent form by the exposure dose of 1 Gy. The results of 6 fluorescent sensors are graphed in Figure 2.

The sensitivities of two analogous dyes, de-esterified $\mathrm{H}_{2}$ DCFDA and DHF, were compared by fluorescence imaging of $100 \mu \mathrm{M}$ solutions as described in the subsection of "Materials and Methods - Initial Selection of Fluorescent ROS Sensors - Fluorescence Microscopy Measurements of Dye Solutions". Figure 3A shows the time course of fluorescence intensity before and during radiation. A linear rise of fluorescence intensity was seen immediately after the $\mathrm{x}$-ray beam was switched on. To confirm that the dye conversion was mediated by free radicals, we also tested de-esterified $\mathrm{H}_{2}$ DCFDA solutions with $1 \mathrm{mM}$ ascorbate (Vitamin $\mathrm{C}$ ) which is a free radical scavenger. The fluorescence level was significantly depressed with the presence of ascorbate. The increase of fluorescence intensity per Gy of radiation for de-

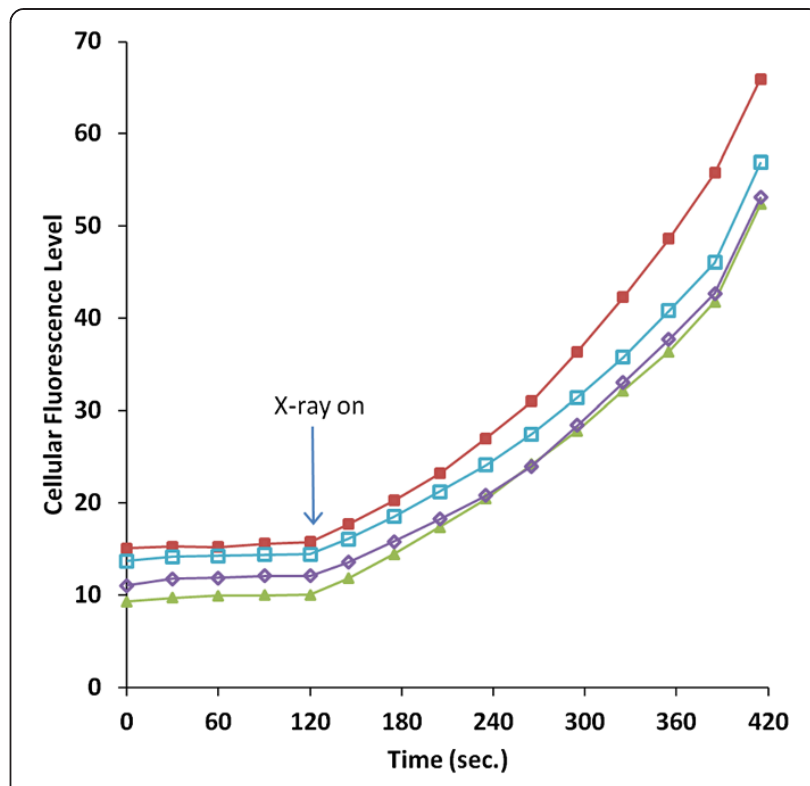

Figure 5 Average cellular fluorescence intensity in MEF cells over the time course of $\mathbf{7}$ minutes. The data from the four experiments are graphed. The Fluorescence intensities were obtained from time series (Additional file 2) taken on the modified confocal microscope (Figure 1) at 30 second intervals. The MEF cells were loaded with the fluorescent ROS sensor $\mathrm{H}_{2} \mathrm{DCFDA}$. The X-ray tube was turned on after the $5^{\text {th }}$ image and stayed on till the end of the series. Cells were kept at room temperature $\left(21^{\circ} \mathrm{C}\right)$ during imaging. The fluorescence intensity was nearly constant before radiation, immediately rose upon onset of the $x$-ray beam, and increased at accelerating pace over the course of radiation. The radiation dose rate was $3.0 \mathrm{~Gy} / \mathrm{min}$.

esterified $\mathrm{H}_{2}$ DCFDA with and without ascorbate and for DHF are graphed in Figure 3B.

From the above results we concluded that among this group of ROS sensors, $\mathrm{H}_{2}$ DCFDA and DHR- 123 had the highest sensitivities to $\mathrm{x}$-ray radiation. However, DHR123 is oxidized to rhodamine 123 upon reaction with ROS, and rhodamine 123 is known to be cationic and
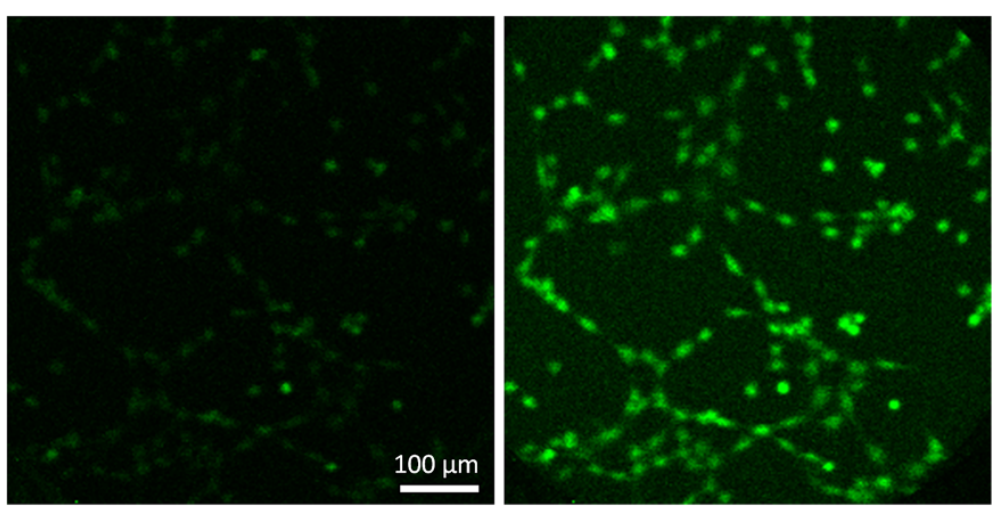

Figure 4 The first and last fluorescence images from a time series of live MEF cells taken before and during $x$-ray radiation. Many cells are visible in the field of view. The images are left) before the $x$-ray beam was switched on; right) at 5 minutes of exposure. The exposure dose rate was $3.0 \mathrm{~Gy} / \mathrm{min}$. 


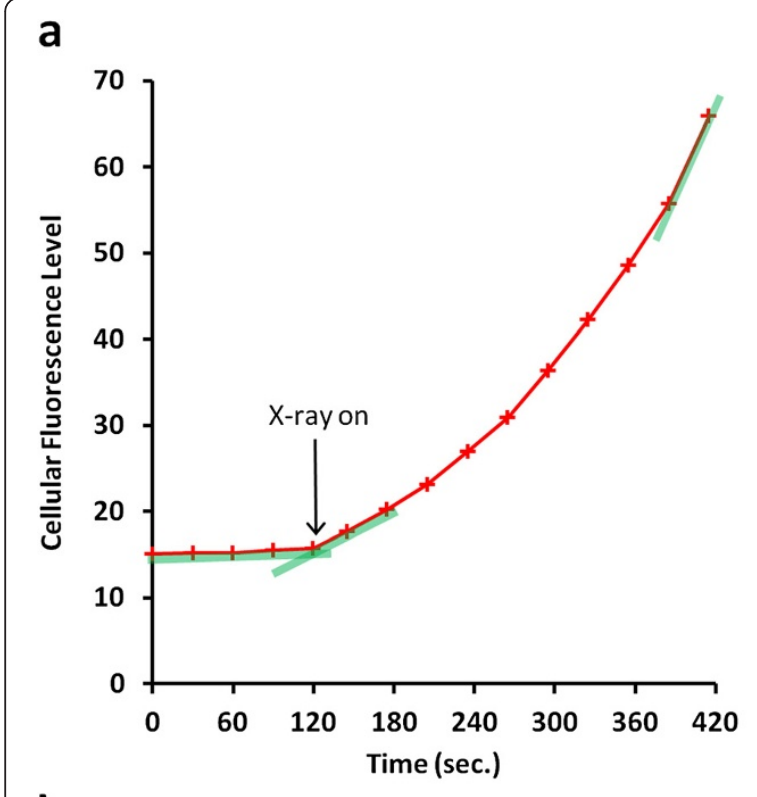

b

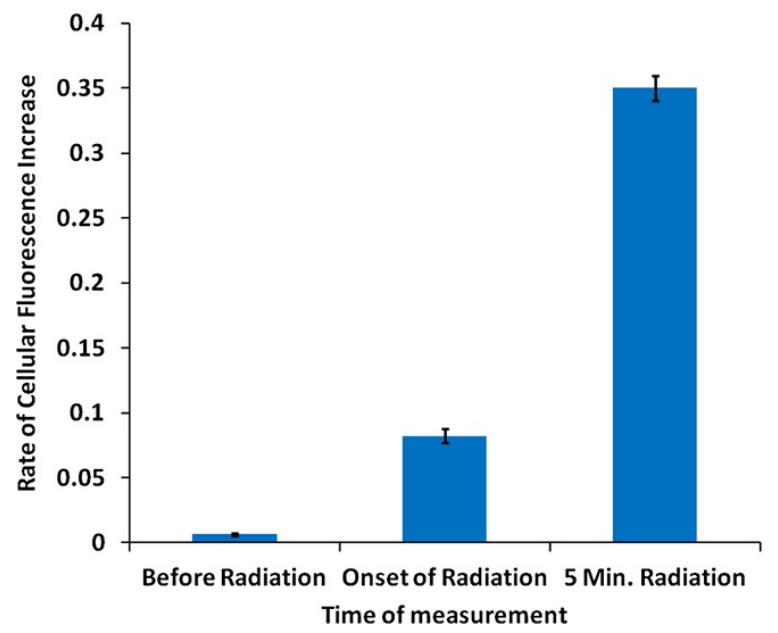

Figure 6 Determination of the rate of increase of cellular fluorescence level at three points in the time series of Figure 5. The time series are confocal fluorescence images acquired before and during $x$-ray radiation (Additional file 2). (a) In a typical time series, the rates of fluorescence increase are illustrated by the green straight segments. The rate prior to the on-switch of the $x$-ray beam was calculated from the slope of the first 5 images. The rate at the onset of $x$-rays was calculated from the increase between the $5^{\text {th }}$ and $6^{\text {th }}$ images. The rate at 5 minutes of radiation was calculated between the $14^{\text {th }}$ and $15^{\text {th }}$ images. (b) The average and standard deviation of the rates of fluorescence increase are graphed for the three time points, among four separate experiments.

collects in mitochondria due to the driving force of membrane potentials. This type of migration and redistribution may introduce changes of fluorescence intensity that are not related to the formation of free radicals. For this reason, $\mathrm{H}_{2} \mathrm{DCFDA}$ was chosen for further cell imaging experiments.
Dynamic imaging of free radical formation in cells under X-Ray

A movie of the time series of fluorescence images over a period of 7 minutes is uploaded as supplemental material. The time period included 2 minutes before the $\mathrm{x}$-ray source was switched on and 5 minutes of $x$-ray irradiation. The fluorescence level in the cells remained low before radiation, and increased steadily during radiation. Representative images before and at the end of the radiation period are shown in Figure 4. The time course of the cellular fluorescence intensity is plotted in Figure 5 for the four separate experiments. The fluorescence intensity was nearly constant before radiation, immediately rose after the on-switch of the $\mathrm{x}$-ray tube at image No. 5, and increased at accelerating pace over the course of radiation.

The rate of increase of the cellular fluorescence intensity at three time points is graphed in Figure 6 . We observed a slightly positive rate of $(6.4 \pm 1.1) \times 10^{-3} \mathrm{sec}^{-1}$ during the 2 minute period before the $\mathrm{x}$-ray was turned on. At the onset of radiation, the initial rate of fluorescence increase varied between $7.5 \times 10^{-2} \mathrm{sec}^{-1}$ and $8.7 \times 10^{-2} \mathrm{sec}^{-1}$ among the 4 experiments, with the average value of $(8.2 \pm 0.5) \times 10^{-2} \mathrm{sec}^{-1}$. At the end of $5 \mathrm{~min}-$ utes of radiation, the rate of increase has risen to 4.3 times the initial rate.

\section{Comparison of cellular fluorescence between X-Ray and UV light exposures}

The total increase in cellular fluorescence intensity from 5 minutes of $\mathrm{x}$-ray exposure was comparable to that

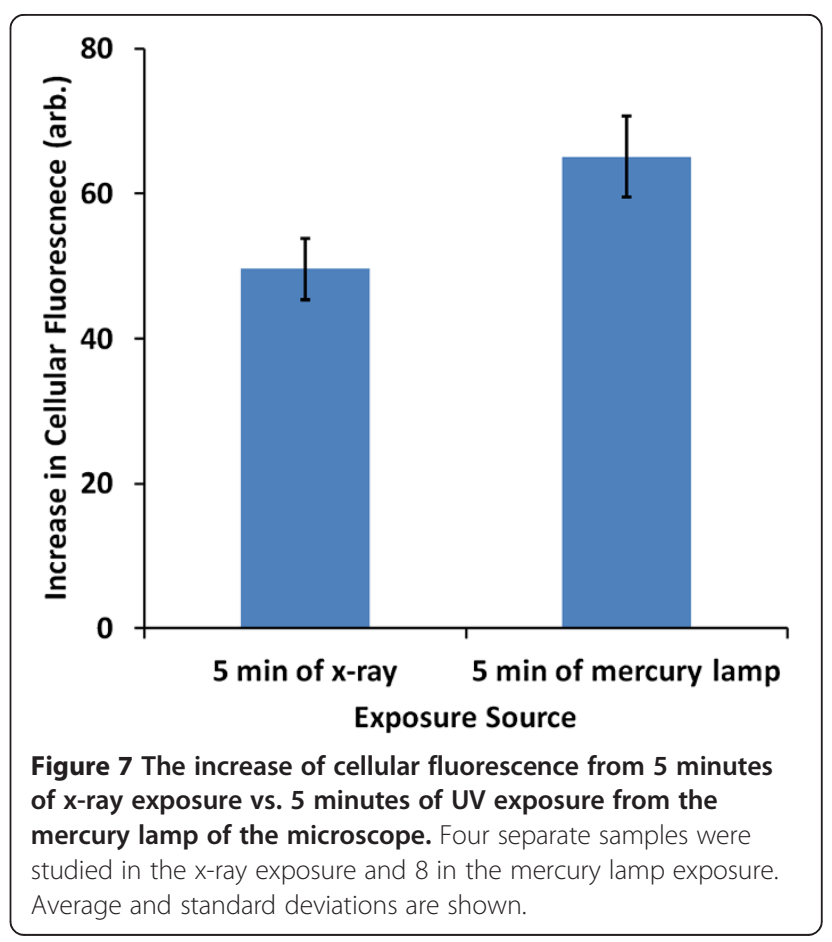


from 5 minutes of UV light exposure using the mercury lamp of the microscope. Figure 7 shows the results for both types of exposure. Based on the mean of both results, the increase of cellular fluorescence from the $x$-ray tube was $76 \%$ of that from the mercury lamp.

\section{Conclusions}

We demonstrated dynamic imaging of the immediate free radical products of $\mathrm{x}$-ray absorption in live cells using the fluorescent ROS sensor $\mathrm{H}_{2}$ DCFDA. We observed a low and near-constant level of fluorescence in the cells without radiation, an initial positive rate of increase at the onset of radiation, and a higher rate of increase at 5 minutes of radiation. These results are consistent with the notion that the initial rate of fluorescence increase corresponds to the immediate free radical products of $\mathrm{x}$-ray ionization events in the cell medium, and the higher rates after a few minutes of radiation includes contributions from both the immediate products of radiolysis and the activation of cellular mechanisms that produce their own reactive oxygen species (Leach et al. 2001; Spitz et al. 2004). The conclusion that we were able to image the initial products of $x$-ray radiolysis was also supported by the fluorescence imaging results in the solutions of the dyes, where biological production is absent.

We showed that several commercially available fluorescent ROS sensors had varying degrees of sensitivity towards of $x$-ray radiation. Among these, DHR-123 and $\mathrm{H}_{2}$ DCFDA had the highest sensitivities. A number of published papers have shown success using $\mathrm{H}_{2}$ DCFDA or its derivatives to detect radiation-induced ROS formation in cells(Leach et al. 2001; Morales et al. 1998; Narayanan et al. 1997; Wan et al. 2003). Our results are consistent with these prior studies.

It is worth noting that due to the low power rating of 20 Watts of our compact $\mathrm{x}$-ray tube, exposure times of many seconds were necessary to accumulate sufficient dose to cause a visible rise in the fluorescence level. The output spectra of tungsten-anode $\mathrm{x}$-ray tubes have been measured by Bhat and co-authors for several generator input voltages(Bhat et al. 1998). At $80 \mathrm{kVp}$ input the spectrum peaks at $32 \mathrm{keV}$ and The spectrum peaks at $32 \mathrm{keV}$ and has a full width at half maximum (FWHM) of $30 \mathrm{keV}$. Knowing the mass absorption coefficient of typical soft tissue at $32 \mathrm{keV}\left(0.34 \mathrm{~cm}^{2} / \mathrm{g}\right)$ and the absorption dose rate at the sample, we estimated the radiation flux density at the sample to be $4.9 \times 10^{-5}$ joule $/ \mathrm{cm}^{2} / \mathrm{sec}$. If we consider that the mass absorption coefficient varies widely over the spectrum of the $\mathrm{x}$-ray tube and is weighted more towards the higher values of lower photon energies, the actual flux density at the sample is likely lower. In comparison, the focused x-ray nanobeams at synchrotron sources and even dedicated laboratory microbeams are $3-10$ orders of magnitude more intense. Therefore, these sources should permit rapid scanning experiments.

For the ultimate purpose of developing a microscopy method based on real-time imaging of the free radical products of a scanning $\mathrm{x}$-ray nanobeam, both $\mathrm{H}_{2}$ DCFDA and DHR-123 are possible candidates. In this context, there are several topics that warrant further research. First, the conversion of the dyes to fluorescent forms by free radicals is irreversible. The advantage of this feature is that transient events such as pulsed $\mathrm{x}$-ray exposures produce permanent effects. However, the disadvantage is that each fluorescent sensor molecule can only be used once. Since the location of a single switching event can be anywhere within the $\mathrm{x}$-ray beam profile, irreversible fluorescent sensors limit the imaging resolution to the size of the $\mathrm{x}$-ray beam plus additional broadening of a few nanometers due to the diffusion of the free radicals. Developments in reversible redox-switched fluorescent proteins (Hanson et al. 2004; Ostergaard et al. 2001) may lead to reversible free radical sensors that are free from this limitation.

Another consideration is that all of the fluorescent dyes we tested are freely diffusible. This allows them to enter the intracellular space, but they are not suitable for labeling specific structures. Future work may include searching for bound forms of free radical sensors in addition to developing the methodology of free radical imaging with a scanning $x$-ray beam.

\section{Additional files}

\section{Additional file 1: Appendix A Preparation of Solutions of Fluorescent ROS Sensors, Appendix B MEF Cell Culture and Handling Protocols. \\ Additional file 2: A movie of the time series of fluorescence images of mouse fibroblast cells before and during $x$-ray irradiation. The cells were pre-loaded with the ROS sensor H2DCFDA.}

\section{Abbreviations}

ROS: Reactive oxygen species; $\mathrm{H}_{2}$ DCFDA: 2',7'-dihydrodichlorofluorescein; DCFH: 2', 7'-dichlorofluorescein; DHR-123: Dihydrorhodamine 123;

DHF: Dihydrofluorescein; APF: Aminophenyl fluorescein; HE: Hydroethidine; Amplex Red: Dihydroresorufin; D-MEM: Dulbecco's Modified Eagle Medium; PBS: Phosphate-buffered saline; DPBS: Dulbecco's phosphate-buffered saline.

\section{Competing interests}

The authors declare they have no competing interests.

\section{Authors' contributions}

C.A.R. performed the experiments and wrote the manuscript. K.M. provided the cell line, participated in cell sample preparation, the experiments, discussions and manuscript editing. H.W. is responsible for the concept and design of the experiments, participated in the experiments and manuscript writing. All authors read and approved the final manuscript.

\section{Acknowledgements}

We thank Dr. Alan Remaley of National Heart, Lung and Blood Institute, National Institutes of Health for assistance with the MEF cell culture, and Dr. Terrence Jach of National Institutes of Standard and Technology for 
additional tests on his $\mathrm{x}$-ray source. We thank Colin Andrews of NHLBI for making the $x$-ray window, and Andrew Gomella of NHLBI for his assistance with graphing. This work was funded by the Division of Intramural Research National Heart, Lung and Blood Institute, National Institutes of Health under project number ZIA HL006141-01.

\section{Author details}

${ }^{1}$ Imaging Physics Lab, Biophysics and Biochemistry Center, National Heart, Lung and Blood Institute, National Institutes of Health, Bethesda, MD 20892, USA. ${ }^{2}$ Laboratory of Cardiac Energetics, Integrative Physiology Center, National Heart, Lung and Blood Institute, National Institutes of Health, Bethesda, MD 20892, USA.

Received: 3 May 2012 Accepted: 11 June 2012

Published: 2 July 2012

\section{References}

Betzig E, Patterson GH, Sougrat R, Lindwasser OW, Olenych S, Bonifacino JS, Davidson MW, Lippincott-Schwartz J, Hess HF (2006) Imaging intracellular fluorescent proteins at nanometer resolution. Science 313:1642-1645

Bhat M, Pattison J, Bibbo G, Caon M (1998) Diagnostic x-ray spectra: A comparison of spectra generated by different computational methods with a measured spectrum. Medical Physics 25:114-120

Cholewa M, Dillon C, Lay P, Phillips D, Talarico T, Lai B (2001) High resolution nuclear and $X$-ray microprobes and their applications in single cell analysis. Nucl Instrum Methods Phys Res, Sect B 181:715-722

Cohn CA, Simon SR, Schoonen MAA (2008) Comparison of fluorescence-based techniques for the quantification of particle-induced hydroxyl radicals. Part Fibre Toxicol 5(2):1-9

Gomes A, Fernandes E, Lima JLFC (2005) Fluorescence probes used for detection of reactive oxygen species. J Biochem Bioph Meth 65:45-80

Hanson GT, Aggeler R, Oglesbee D, Cannon M, Capaldi RA, Tsien RY, Remington SJ (2004) Investigating mitochondrial redox potential with redox-sensitive green fluorescent protein indicators. J Biol Chem 279:13044-13053

Ice GE, Budai JD, Pang JWL (2011) The Race to X-ray Microbeam and Nanobeam Science. Science 334:1234-1239

Kang HC, Maser J, Stephenson GB, Liu C, Conley R, Macrander AT, Vogt S (2006) Nanometer linear focusing of hard $x$ rays by a multilayer Laue lens. Phys Rev Lett 96(12):127410

Kim E-H (2008) Better Understanding of the Biological Effects of Radiation by Microscopic Approaches. Nuclear Engineering and Technology 40:551-560

Kneip S, McGuffey C, Martins JL, Martins SF, Bellei C, Chvykov V, Dollar F, Fonseca R, Huntington C, Kalintchenko G et al (2010) Bright spatially coherent synchrotron $X$-rays from a table-top source. Nat Phys 6:980-983

Leach JK, Van Tuyle G, Lin PS, Schmidt-Ullrich R, Mikkelsen RB (2001) lonizing radiation-induced, mitochondria-dependent generation of reactive oxygen/ nitrogen. Cancer Res 61:3894-3901

Meesungnoen J, Benrahmoune M, Filali-Mouhim A, Mankhetkorn S, Jay-Gerin JP (2001) Monte Carlo calculation of the primary radical and molecular yields of liquid water radiolysis in the linear energy transfer range 0.3-6.5 keV/mu m: Application to Cs-137 gamma rays (vol 155, pg 269, 2001). Radiat Res 155:873

Mimura H, Handa S, Kimura T, Yumoto H, Yamakawa D, Yokoyama H, Matsuyama S, Inagaki K, Yamamura K, Sano Y et al (2010) Breaking the $10 \mathrm{~nm}$ barrier in hard $x$-ray focusing. Nat Phys 6:57-60

Morales A, Miranda M, Sanchez-Reyes A, Biete A, Fernandez-Checa JC (1998) Oxidative damage of mitochondrial and nuclear DNA induced by ionizing radiation in human hepatoblastoma cells. Int J Radiat Oncol Biol Phys 42:191-203

Narayanan PK, Goodwin EH, Lehnert BE (1997) alpha particles initiate biological production of superoxide anions and hydrogen peroxide in human cells. Cancer Res 57:3963-3971

Ostergaard H, Henriksen A, Hansen FG, Winther JR (2001) Shedding light on disulfide bond formation: engineering a redox switch in green fluorescent protein. EMBO J 20:5853-5862

Patterson GH, Lippincott-Schwartz J (2002) A photoactivatable GFP for selective photolabeling of proteins and cells. Science 297:1873-1877

Prise KM, Schettino G (2011) Microbeams in Radiation Biology: Review and Critical Comparison. Radiat Prot Dosimetry 143:335-339
Roots R, Okada S (1975) Estimation of Life Times and Diffusion Distances of Radicals Involved in X-Ray-Induced DNA Strand Breaks or Killing of Mammalian-Cells. Radiat Res 64:306-320

Schmidt-Ullrich RK, Dent P, Grant S, Mikkelsen RB, Valerie K (2000) Signal transduction and cellular radiation responses. Radiat Res 153:245-257

Sehested K, Bjergbak E, Fricke H (1973) Primary Species Yields in Co-60 Gamma-Ray Radiolysis of Aqueous-Solutions of $\mathrm{H} 2$ so 4 between Ph 7 and 0.46. Radiat Res 53:385-399

Spitz DR, Azzam El, Li JJ, Gius D (2004) Metabolic oxidation/reduction reactions and cellular responses to ionizing radiation: A unifying concept in stress response biology. Cancer Metastasis Rev 23:311-322

Wan XS, Zhou ZZ, Kennedy AR (2003) Adaptation of the dichlorofluorescein assay for detection of radiation-induced oxidative stress in cultured cells. Radiat Res 160:622-630

Wardman P (2007) Fluorescent and luminescent probes for measurement of oxidative and nitrosative species in cells and tissues: Progress, pitfalls, and prospects. Free Radical Bio Med 43:995-1022

doi:10.1186/2192-2853-1-5

Cite this article as: Rappole et al.: Dynamic fluorescence imaging of the free radical products of X-ray absorption in live cells. Optical Nanoscopy 2012 1:5.

\section{Submit your manuscript to a SpringerOpen ${ }^{\circ}$ journal and benefit from:}

- Convenient online submission

- Rigorous peer review

- Immediate publication on acceptance

- Open access: articles freely available online

- High visibility within the field

- Retaining the copyright to your article

Submit your next manuscript at $\gg$ springeropen.com 